Category: Original Article

\title{
Effect of preterm birth on growth and cardiovascular disease risk at school age
}

Short title: Preterm birth at school age

Satomi Inomata ${ }^{1}$, Taketoshi Yoshida ${ }^{1 *}$, Uta Koura ${ }^{1}$, Kentaro Tamura ${ }^{1}$, Kiyoshi Hatasaki ${ }^{2}$, Hiroaki Imamura ${ }^{3}$, Daisuke Mase ${ }^{4}$, Mika Kigawa ${ }^{5}$, Yuichi Adachi ${ }^{6}$, Hidekuni Inadera ${ }^{5}$

${ }^{1}$ Division of Neonatology, Maternal and Perinatal Center, Toyama University Hospital, Toyama, Japan; ${ }^{2}$ Toyama Prefectural Central Hospital, Toyama, Japan; ${ }^{3}$ Koseiren Takaoka Hospital, Toyama, Japan; ${ }^{4}$ Toyama City Medical Association, Toyama, Japan; ${ }^{5}$ Department of Public Health, Faculty of Medicine, University of Toyama, Toyama, Japan; ${ }^{6}$ Department of Pediatrics, Faculty of Medicine, University of Toyama, Toyama, Japan

\section{Correspondence:}

Taketoshi Yoshida, MD, PhD

Division of Neonatology, Maternal and Perinatal Center, Toyama University Hospital, 2630

Sugitani, Toyama 930-0194, Japan

Tel: +81-76-434-7313

Fax: +81-76-434-5029

E-mail: ytake@med.u-toyama.ac.jp

Number of text pages: 9, Reference pages: 3, Tables: 3 


\begin{abstract}
Background. Low birth weight is associated with increased risk for cardiovascular disease (CVD) in later life. However, whether premature birth is also a risk factor for CVD has not been fully determined. The aim of this study was to investigate the relationship between gestational age and risk factors for CVD at school age.

Methods. Using medical checkup data of school children, the relationship between gestational age and height, weight, body mass index, blood pressure, and lipid profiles at ages 9 and 12 years were investigated in children born preterm and admitted to neonatal intensive care unit at birth ( $\mathrm{n}=182 ; 115$ boys and 67 girls). These data were also compared between preterm small for gestational age (SGA) children and preterm appropriate for gestational age (AGA) children.

Results. Gestational age was positively associated with height, and inversely associated with systolic blood pressure at school age. Preterm SGA children were significantly shorter and lighter at 9 and 12 years of age compared with preterm AGA children. However, there were no significant differences in any CVD risk factors between the groups.

Conclusions. In preterm infants, a shorter duration of gestation is associated with higher systolic blood pressure at school age.

(196 words).
\end{abstract}

Keywords: premature birth, blood pressure, medical checkup, growth, newborns 
The survival of preterm infants has significantly improved, largely as a result of advances in neonatal care, and an increasing number of these children reach adulthood. To date, many reports have focused on the growth and psychomotor development of preterm infants. Several epidemiological studies have indicated that low birth weight (LBW) is associated with a higher incidence of death from cardiovascular disease (CVD) in later life [1-4]. In addition, LBW is correlated with CVD, as well as type 2 diabetes, dyslipidemia, and hypertension in adulthood [5]. These studies have led to the hypothesis that prenatal and early postnatal environment permanently changes the body's structure, function, and metabolism, and predisposes individuals to CVD in later life [6]. Although there are many reports indicating the relationship of LBW to later diseases, only a few studies have investigated the relationship between preterm infants and CVD risk factors at school age. Most previous studies focused on subjects who were born small for gestational age (SGA) at term, or included only a few subjects who were born preterm. Thus, these results did not necessarily reflect the state of preterm infants.

Although long-term follow up is important for preterm infants who were previously hospitalized in a neonatal intensive care unit (NICU), it is difficult to follow these children to adulthood. However, medical checkups for school children are routinely conducted in the Toyama Prefecture, Japan, and include anthropometric measurements, lipid concentrations, and blood pressure. Here, we analyzed medical checkup data of school children who had been hospitalized in NICUs at birth, to investigate the relationship of preterm birth on CVD risk factors at school age. The aim of the current study was to determine whether prematurity or SGA are associated with CVD risk factors at school age.

\section{Methods}

This study was a retrospective cohort study. Medical checkups for school children at 
the age of 9-10 years and 12-13 years have been routinely conducted to screen for lifestyle-related diseases in Toyama and Takaoka cities of the Toyama Prefecture, Japan, by the area's local governments. Although these checkups are voluntary for school children, over $90 \%$ of school children participated in the medical checkups. In this study, we recruited school children who were born preterm ( $<37$ completed weeks' gestation) and admitted to the NICU of Toyama University Hospital during April 1990 to March 2001, or the NICUs of Toyama Prefectural Central Hospital or Koseiren Takaoka Hospital during April 1994 to March 1999. These three hospitals are capable of caring for most preterm infants in the Toyama Prefecture. Based on hospital records, we excluded patients who were not preterm, did not survive, or who had a history of congenital heart diseases, chromosomal abnormalities, or congenital abdominal diseases. Using the postal system, written informed consent was obtained from the patients' parents regarding the use of perinatal data and health checkup data at school age. After they agreed to use of medical checkup data, we presented their consent forms to the Toyama City Medical Association and the Takaoka City Board of Education that contained all medical checkup data. For the protection of personal information, we anonymized the data after integration of perinatal and medical checkup data. Ethical approval was obtained from the ethics committees of each hospital.

We obtained data of clinical characteristics at birth, including gestational age and birth weight using hospital records. Birth weight was recorded to the nearest $1 \mathrm{~g}$. In our study, SGA was defined as birth weight <10th percentile for gestational age, and appropriate for gestational age (AGA) was defined as $\geq 10$ th percentile and $\leq 90$ th percentile for gestational age based on reference standards of Japanese neonates [7].

Medical checkups were carried out at 9 or 12 years old at the beginning of the school year. Height, weight, blood pressure, serum total cholesterol (TC), high-density lipoprotein cholesterol (HDL-C), low-density lipoprotein cholesterol (LDL-C), triglyceride (TG), glutamic pyruvic transaminase (GPT) levels, and blood count were measured. Children were 
instructed to eat a lighter breakfast than usual. Therefore, blood samples were obtained from the non-fasting state in the morning. Height was recorded to the nearest $0.1 \mathrm{~cm}$ and weight was recorded to the nearest $100 \mathrm{~g}$. Routine analytical methods were used to determine serum concentrations of TC, HDL-C, TG, and GPT. LDL-C was estimated using Friedewald's formula (LDL-C = TC - HDL-C - TG/5). Body mass index (BMI) was calculated using the formula $\left(\right.$ BMI $=$ weight $[\mathrm{kg}] /$ height $\left.[\mathrm{m}]^{2}\right)$. All data were stored at the Toyama City Medical Association or the Takaoka City Board of Education.We investigated the relationships between gestational age and medical checkup data at school age. We adjusted for confounding factors (sex, weight at the time of checkup, and SGA) using multiple regression analysis. Associations between anthropometric values and gestational age were adjusted only for sex and SGA, because correlations between anthropometrical values and weight at the time of measurement were evident. The values of weight, BMI, HDL-C, TG at 9-10 years, weight, BMI, systolic blood pressure, LDL-C, and TG at 12-13 years were log-transformed before analyses because of skewed distributions. These data were also compared between SGA children (subjects who were born SGA) and AGA children (subjects who were born AGA or heavier). We adjusted for confounding factors (sex, weight at the time of checkup, and gestational age) using multiple regression analysis. To evaluate potential selection bias, we compared perinatal data (birth weight, gestational age) of the responders (subjects who agreed to participate in this study) and non-responders (subjects who did not agree to participate in this study, or when we could not obtain data at school age), using the Wilcoxon rank sum test or the chi-square test. Data were analyzed using JMP version 9 (SAS Institute Inc., Cary, NC, USA). A p value of $<0.05$ was considered statistically significant.

\section{Results}

A total of 2314 subjects were admitted to three NICUs during the study period. We 
excluded 1850 subjects because of term infants, their place of residence where blood sampling was not conducted at school age, and history of congenital diseases. We sent a letter to 464 subjects. A total of 138 letters were undeliverable and 121 letters were unanswered. A total of 199 subjects agreed to participate. We excluded 17 subjects who agreed to participate because they had no medical checkups at the age of 9-10 years or at the age of 12-13 years. The total study population comprised 182 subjects (115 boys and 67 girls).

Clinical characteristics at birth are shown in Table 1. A total of 164 subjects (90\%) were LBW infants (birth weight, $<2500$ g), 13 subjects (7.1 \%) were extremely low birth weight infants (birth weight, <1000 g), and 35 subjects (19.2 \%) were very low birth weight infants (birth weight, 1000-1500 g). Fifty subjects were SGA, 131 subjects were AGA or heavier, and 1 subject was unknown. A total of 170 subjects (108 boys and 62 girls) received medical checkups at 9-10 years old, and 107 subjects (69 boys and 38 girls) received them at 12-13 years old. The gestational age of non-responders was $32.7 \pm 2.87$ weeks (responders : $33.4 \pm 3.13$ weeks), birth weight was $1801 \pm 524$ g (responders: $1844 \pm 542$ g). Gestational age and birth weight were not significantly different between the responders and non-responders.

In multiple regression analyses, at the age of 9-10 years, gestational age was positively associated with height (Table 2, $\mathrm{p}=0.01$ ); however, there was no independent association with other anthropometric values (Table 2). Gestational age was also inversely associated with systolic blood pressure $(\mathrm{p}=0.04)$. However, there was no independent association between gestational age and diastolic pressure, or lipid levels at the age of 9-10 years (Table 2). At the age of 12-13 years, gestational age was also positively associated with height (Table 3, $\mathrm{p}=0.01$ ) and weight $(\mathrm{p}=0.02)$. Gestational age was also inversely associated with systolic blood pressure ( $\mathrm{p}=0.02)$. There was no independent association between gestational age and diastolic pressure, or lipid levels at the age of 12-13 years (Table 2).

SGA children were significantly shorter and lighter than AGA children at ages of 9- 
10 years (height, $\mathrm{p}=0.009$; weight, $\mathrm{p}=0.007$ ) and $12-13$ years (height, $\mathrm{p}=0.009$; weight, $\mathrm{p}=0.008$; Table 3). However, there were no significant differences in BMI, blood pressure, or lipid levels between the two groups.

\section{Discussion}

In this study, we obtained two important clinical findings. First, in preterm born children, gestational age was positively associated with height, and inversely associated with systolic blood pressure at school age. Second, SGA born preterm children were significantly shorter and lighter compared with AGA born preterm children at school age. However, there were no significant differences in any CVD risk factors at school age between the groups.

In preterm born children, gestational age was positively associated with height, and inversely associated with systolic blood pressure at school age. In other words, premature infants were smaller, but with higher systolic blood pressure at school age. Although children's blood pressure is affected by body size [8], our data show an inverse correlation between blood pressure and height. This finding is consistent with previous reports, which suggest premature birth can affect blood pressure in later life $[9,10]$. In contrast, some authors insist post-natal factors can have a profound effect on blood pressure compared with prematurity $[11,12]$. There are several reasons for these contradictory results. Blood pressure is influenced by several factors including age, body weight, weight gain during childhood and adolescence, and socio-economic status $[8,11,12]$.

Barker et al. showed that LBW was related to an increased risk of hypertension in adulthood [1,13]. Based on our results, preterm birth could also be a risk factor for hypertension in later life. Specifically, nephrogenesis is completed within 36 weeks' gestation in humans, and the number of nephrons is reduced in preterm infants, which could result in hypertension at school age [14]. However, preterm birth before completion of nephrogenesis did not lead to a reduction in nephron number in a baboon model of preterm birth [15]. Thus, 
further studies are needed to clarify the exact mechanisms underlying preterm birth and risk of developing higher blood pressure in later life. Because higher blood pressure is one of the components of metabolic syndrome, our result suggests that prematurity may increase the risk of CVD.

In this study, we also found that preterm SGA children were significantly shorter and lighter at the ages of 9-10 and 12-13 years compared with preterm AGA children, which is consistent with previous reports [16-18]. However, we did not find any differences in CVD risk factors between the groups. Cheung et al. reported that children born preterm SGA had higher systolic blood pressure compared with children born preterm AGA at 8 years old [19]. In addition, Hofmann et al. showed that preterm birth itself was a risk factor for insulin resistance in childhood, regardless of whether infants were born SGA or AGA [20]. Therefore, preterm birth may be a risk factor for CVD such as insulin resistance. The contradictory results concerning blood pressure require further investigation to clarify the exact effect of preterm birth on blood pressure in adulthood.

In contrast to our expectations, gestational age was not associated with lipid levels at school age. Previous studies have reported that small body size at birth is associated with unfavorable lipid profiles [21-24]. These studies included few preterm infants because most of them were term SGA infants. Only a few investigators have studied the association between preterm infants and lipid levels in later life [25,26]. Interestingly, one study reported that preterm birth was associated with greater total fat mass, but relatively favorable lipid profile in early adulthood [26]. Thus, preterm birth may affect cholesterol metabolism in the long term, but its effect on cholesterol concentration is still controversial. Because plasma cholesterol levels increase and LDL catabolism decreases with age [27], further study for a longer follow-up period and with a larger cohort is required to determine whether prematurity is a cause of dyslipidemia in later life.

There are several limitations of the current study. First, we did not examine physical 
activity levels, diet, socio-economic status, or the family's history of CVD. These factors are strongly correlated with CVD risk factors in later life. Although social status can affect the results, a previous study showed that social status within children born preterm was not related to cholesterol metabolism [28]. Although parental size is reported to be an important predictor of the height of children [26,28], we did not investigate parental height. We only analyzed data according to gestational age. In addition, the pubertal stage of the study subjects could not be defined. Pubertal status might have influenced the biochemical parameters assessed in this study. Finally, we could not obtain a blood sample in the fasting state. TG is known to be affected by meals. Therefore, there might have been bias that was associated with eating behavior.

In conclusion, we found that preterm birth is associated with shorter height and higher systolic blood pressure at school age. An association between preterm birth and unfavorable lipid profiles was not demonstrated in this study. SGA children do not show any risk factors for CVD at school age compared with AGA children. Our results suggest that medical checkups at school age are useful for the early detection of CVD risk factors. Further research is necessary to analyze prenatal and postnatal environmental factors, which determine pathological outcomes, including risk factors for CVD in later life.

\section{Acknowledgments}

This study was supported in part by the Takeda Science Foundation. We thank the staff of the Toyama City Medical Association and the Takaoka City Board of Education for kindly collecting medical checkup data.

\section{Declaration of Interest}

The authors report no declarations of interest. 


\section{References}

1. Barker DJ, Winter PD, Osmond C, Margetts B, Simmonds SJ. Weight in infancy and death from ischaemic heart disease. Lancet 1989;2:577-80.

2. Rich-Edwards JW, Stampfer MJ, Manson JE, et al. Birth weight and risk of cardiovascular disease in a cohort of women followed up since 1976. BMJ 1997;315:396-400.

3. Leon DA, Lithell HO, Vâgerö D, et al. Reduced fetal growth rate and increased risk of death from ischaemic heart disease: cohort study of 15000 Swedish men and women born 1915-29. BMJ 1998;317:241-5.

4. Huxley R, Owen CG, Whincup PH, et al. Is birth weight a risk factor for ischemic heart disease in later life? Am J Clin Nutr 2007;85:1244-50.

5. de Boo HA, Harding JE. The developmental origins of adult disease (Barker) hypothesis. Aust. NZ J. Obstet. Gynaecol 2006;46:4-14.

6. Inadera H. Developmental origins of obesity and type 2 diabetes: molecular aspects and role of chemicals. Environ Health Prev Med 2013;18:185-97.

7. Ogawa Y, Iwamura T, Kuriya N. Birth size standards by gestational age for Japanese neonates. Acta Neonatal Jpn 1998;34:624-32. (in Japanese)

8. Salgado CM, Jardim PC, Teles FB, Nunes MC. Influence of low birth weight on microalbuminuria and blood pressure of school children. Clin Nephrol 2009;71:367-74.

9. Irving RJ, Belton NR, Elton RA, Walker BR. Adult cardiovascular risk factors in premature babies. Lancet 2000;355:2135-6.

10. Rotteveel J, van Weissenbruch MM, Twisk JW, Delemarre-Van de Waal HA. Infant and childhood growth patterns, insulin sensitivity, and blood pressure in prematurely born young adults. Pediatrics 2008;122:313-21.

11. Burke V, Beilin LJ, Blake KV, et al. Indicators of fetal growth do not independently predict blood pressure in 8-year-old Australians: a prospective cohort study. Hypertension 2004;43:208-13. 
12. Falkner B, Hulman S, Kushner H. Effect of birth weight on blood pressure and body size in early adolescence. Hypertension 2004;43:203-7.

13. de Jong F, Monuteaux MC, van Elburg RM, Gillman MW, Belfort MB. Systematic review and meta-analysis of preterm birth and later systolic blood pressure. Hypertension 2012;59:226-34.

14. Bertram JF, Douglas-Denton RN, Diouf B, Hughson MD, Hoy WE. Human nephron number: implications for health and disease. Pediatr Nephrol 2011;26:1529-33.

15. Sutherland MR, Gubhaju L, Black MJ. Stereological assessment of renal development in a baboon model of preterm birth. Am J Nephrol 2011;33:25-33.

16. Karlberg J, Albertsson-Wikland K. Growth in full-term small-for-gestational-age infants: from birth to final height. Pediatr Res 1995;38:733-9.

17. Leger J, Levy-Marchal C, Bloch J, et al. Reduced final height and indications for insulin resistance in 20 year olds born small for gestational age: regional cohort study. BMJ 1997;315:341-7.

18. Simon D, Léger J, Carel JC. Optimal use of growth hormone therapy for maximizing adult height in children born small for gestational age. Best Pract Res Clin Endocrinol Metab 2008;22:525-37.

19. Cheung YF, Wong KY, Lam BC, Tsoi NS. Relation of arterial stiffness with gestational age and birth weight. Arch Dis Child 2004;89:217-21.

20. Hofman PL, Regan F, Jackson WE, et al. Premature birth and later insulin resistance. N Engl J Med 2004;351:2179-86

21. Owen CG, Whincup PH, Odoki K, Gilg JA, Cook DG. Birth weight and blood cholesterol level: a study in adolescents and systematic review. Pediatrics 2003;111:1081-9.

22. Huxley R, Owen CG, Whincup PH, et al. Birth weight and subsequent cholesterol levels: exploration of the "fetal origins" hypothesis. JAMA 2004;292:2755-64. 
23. Kaneshi T, Yoshida T, Ohshiro T, et al. Birth weight and risk factors for cardiovascular diseases in Japanese school children. Pediatr Int 2007;49:138-43.

24. Salonen M, Tenhola S, Laitinen T, et al. Tracking serum lipid levels and the association of cholesterol concentrations, blood pressure and cigarette smoking with carotid artery intima-media thickness in young adults born small for gestational age. Circ $\mathrm{J}$ 2010;74:2419-25.

25. Mortaz M, Fewtrell MS, Cole TJ, Lucas A. Cholesterol metabolism in 8 to 12-year-old children born preterm or at term. Acta Paediatr 2003;92:525-30.

26. Breukhoven PE, Kerkhof GF, Willemsen RH, Hokken-Koelega AC. Fat mass and lipid profile in young adults born preterm. J Clin Endocrinol Metab 2012;97:1294-302.

27. Desai M, Hales CN. Role of fetal and infant growth in programming metabolism in later life. Biol Rev Camb Philos Soc 1997;72:329-48.

28. Mortaz M, Fewtrell MS, Cole TJ, Lucas A. Birth weight, subsequent growth, and cholesterol metabolism in children 8-12 years old born preterm. Arch Dis Child 2001;84:212-7. 